\title{
Implementación de un sistema de evaluación para mejorar el rendimiento académico en la asignatura de matemática, en la Universidad Nacional «Santiago Antúnez de Mayolo»
}

Implementation of an evaluation system to improve academic performance in Mathematics, at the National University "Santiago Antunez de Mayolo"

${ }^{1}$ Cesar A. González Chiclayo ${ }^{\mathrm{a}},{ }^{1}$ Eleuterio Ramírez Apolinario ${ }^{\mathrm{b}}$, ${ }^{1}$ María L. Medina Gutierres ${ }^{\mathrm{c}}$, ${ }^{1}$ Merlyn Zavaleta Medina ${ }^{\mathrm{a}}$

\section{RESUMEN}

Se a realizado un estudio de la influencia que una forma de evaluación denominado Evaluación Sistemática, puede tener en el rendimiento y el aprendizaje de los estudiantes de Matemática I en la Universidad Nacional "Santiago Antúnez de Mayolo". La población objeto de estudio estuvo conformado por alumnos de Matemática de las Escuelas Profesionales de Ingeniería Agrícola y Agronomía matriculados en el Semestre 2015-I. La investigación es de tipo Cuasi experimental. Para la contrastación de hipótesis se utilizó el diseño de dos grupos: Grupo experimental y grupo control. Ambos estuvieron sujetos a un tipo característico de evaluación denominado sistemática y tradicional respectivamente. Para el análisis de medias se ha utilizado la prueba estadística t-Student con un nivel de significancia al 5\% y para el análisis de varianzas se ha utilizado la prueba de Levene. Los resultados confirman que la aplicación de una evaluación correctamente sistematizada influye significativamente en el aprendizaje y rendimiento académico de los estudiantes.

Palabras clave: Evaluación; aprendizaje; instrumentos de evaluación

\begin{abstract}
A study was made of the influence that a form of evaluation called Systematic Evaluation can have on the performance and learning of Mathematics I students at the National University "Santiago Antúnez de Mayolo". The population studied was made up of Mathematics I students of the Professional Schools of Agricultural Engineering and Agronomy enrolled in the Semester 2015-I. The research is of the quasi-experimental type. For the hypothesis testing, the design of two groups was used: experimental group and control group. Both were subject to a characteristic type of evaluation called systematic and traditional respectively. For the analysis of means, the t-Student statistical test with a level of significance of $5 \%$ was used, and for the analysis of variances the Levene test was used. The results confirm that the application of a correctly systematized evaluation significantly influences the learning and academic performance of the students.
\end{abstract}

keyword: Evaluation; learning; evaluation instruments

${ }^{1}$ Universidad Nacional Santiago Antúnez de Mayolo Huaraz, Perú. Lic. en matemática.

${ }^{\mathrm{b}}$ Lic. en Física. ${ }^{\mathrm{c}}$ Lic. en estadística. 


\section{INTRODUCCIÓN}

La evaluación es un componente inseparable del proceso enseñanzaaprendizaje, pues permanentemente los profesores, según sus concepciones $\mathrm{y}$ creencias, afectan con sus decisiones (algunas veces irreversibles), los intereses, las actitudes y las expectativas de formación de generaciones de jóvenes que se educan en los diversos programas educativos. Según, Antoni (2008) evaluar no es una tarea fácil, el docente necesita utilizar técnicas e instrumentos que le proporcionen la información necesaria para establecer un diagnostico previo, para desarrollar y completar el proceso evaluador, lo cual implica conocer un amplio abanico de posibilidades en ese ámbito. En la práctica educativa, una buena información acerca del aprendizaje en los alumnos, está asociada al buen uso de los instrumentos e indicadores de evaluación, que pretenden diagnosticar cuanto ha aprendido el alumno. Moreno (2016) sostiene que, si la información que arroja la evaluación sirve al profesor para mejorar su propia enseñanza, y reorientar y ayudar a los alumnos en su aprendizaje, éste puede desempeñar un papel constructivo en la formación del alumno. Sin embargo, Moya (2013), indica que muchos docentes adolecen de formación en evaluación y sobre todo, les falta preparación en la elaboración de instrumentos de evaluación que sirvan como diagnostico de lo aprendido y a su vez, como herramientas de retroalimentación en el proceso del aprendizaje. Esta aseveración se acerca mucho a nuestra realidad universitaria, más si tomamos en cuenta, que el docente de Matemáticas en la Universidad Santiago Antúnez de Mayolo (UNASAM) tiene poca o nula formación pedagógica. La escasez de información acerca de la influencia que la evaluación puede tener en el aprendizaje de nuestros estudiantes, se hace más evidente aún al constatar en nuestra experiencia, la forma empírica como es aplicado el proceso de evaluación con los estudiantes, que pasa por proponer el desarrollo de preguntas y su respectiva respuesta; sobre temas desarrollados previamente. Sin identificar las insuficiencias individuales y la aplicación de correcciones y ajustes que regulen el curso del aprendizaje, como lo sostiene Skovsmose (2012). Por ello el objetivo del presente estudio es la de establecer el vinculo que existe entre una buena planificación de evaluación y el incremento del aprendizaje y el rendimiento de los estudiantes en la asignatura de Matemática I.

\section{MATERIALES Y MÉTODOS}

La población accesible estuvo constituido por alumnos de la asignatura de Matemática de las Escuelas Profesionales de la Universidad Nacional «Santiago Antúnez de Mayolo», matriculados en el semestre 2015-I. Distribuidos de la siguiente manera: 


\begin{tabular}{lc} 
ESCUELA PROFESIONAL & $\mathrm{N}^{\circ}$ DE ALUMNOS \\
\hline Ingeniería Agrícola & 25 \\
Agronomía & 25
\end{tabular}

El presente estudio se realizo considerando a los alumnos de la asignatura de Matemática, de las Escuelas Profesionales de Ingeniería Agrícola y de Agronomía, que asistieron regularmente en el desarrollo de la Asignatura de Matemática, durante el semestre 2015-I; los cuales fueron distribuidos en dos grupos, de acuerdo a lo siguiente:

\begin{tabular}{lcc} 
Grupo & $\mathrm{N}^{\circ}$ de Alumnos & Escuela Profesional \\
\hline Control & 25 & Agronomía \\
Experimental & 25 & Ingeniería Agrícola \\
\hline
\end{tabular}

\section{Grupo Control}

Formado por los alumnos de la Escuela profesional de Agronomía, fueron evaluados de forma tradicional, como acostumbran hacerlo los docentes de las Asignaturas de Matemáticas; es decir, mediante la aplicación de cuatro prácticas calificadas y dos exámenes parciales, previo a los exámenes se realiza seminarios de ejercicios.

\section{Grupo Experimental}

Formado por los alumnos de la Escuela Profesional de Ingeniería Agrícola, en donde el docente estableció un tipo de evaluación personalizado, tomando las mismas evaluaciones que el grupo control, pero identificando mediante instrumentos de seguimiento y evaluación, las deficiencias de aprendizaje de los alumnos y ayudado por estos instrumentos, realizó retroalime- ntación en horas de asesoría y tutoría.

\section{Diseño De Investigación}

El diseño de investigación utilizado fue el cuasi experimental, por la imposibilidad de la experimentación rigurosa, ya que los sujetos de los grupos de estudio (control y experimental), no son asignados aleatoriamente. El tipo de diseño cuasi. Experimental fue el de series temporales que implica, según Arias (2012), realizar medición de variables dependientes en los grupos de estudio, antes de la aplicación de la variable independiente y luego de dicha aplicación realizar una nueva medición de la variable de interés; es decir, significa aplicar series de preprueba y pos-prueba.

\section{Instrumentos}

Para la recolección de información se utilizó los siguientes instrumentos:

Prácticas calificadas: cuatro en total, dos antes del examen parcial y dos antes del examen final. Sirvió para obtener información de los logros obtenidos a mitad de semestre y al final.

Matriz de datos: para incorporar los resultados de las prácticas calificados, a fin de establecer los avances y dificultades de cada uno de los alumnos.

Tabla de especificaciones: para los exámenes parcial y final. A fin de establecer los indicadores de logro, relacionados con los objetivos propuestos en el plan de estudios. 


\section{Metodología}

La recolección de información se inicio con la aplicación con la aplicación de las prácticas calificadas a los alumnos de ambos grupos. En el grupo experimental sirvió para identificar debilidades y progreso de cada estudiante, con el propósito de reforzamiento. Con los resultados de los exámenes (parcial y final), se estableció una distribución normal de la población utilizando la prueba de Kolgomorov- Smirrnov , luego se halló las medias estadísticas (media, varianza), que permitieron analizar el comportamiento de la información. Además, con estas mismas medias estadísticas se contrastan la hipótesis utilizando una distribución que para el siguiente caso, por ser una población pequeña, se aplico la distribución t.de Student. Para comprobar si existen diferencias de varianzas entre las medias de los exámenes se utilizó la prueba de Levene de homogeneidad de Varianzas, ya que se cumpla o no, ésta condición dependerá de la formulación que se emplee en el contraste de hipótesis.

\section{RESULTADOS}

Tabla 1. Prueba de Kolgomorov - Smirnov, para establecer la distribución normal de la población

$\begin{array}{cccccl}\text { GC } & \text { GC } & \text { GE } & \text { GE } & \text { GC } & \text { GE } \\ \text { Ex. } & \text { Ex. } & \text { Ex. } & \text { Ex. } & \text { Ex. } & \text { Ex. } \\ \text { Parcial } & \text { Final } & \text { Parcial } & \text { Final } & \text { Entrada } & \text { Entrada }\end{array}$

\begin{tabular}{|c|c|c|c|c|c|c|c|}
\hline & $\mathrm{N}$ & 25 & 25 & 25 & 25 & 25 & 25 \\
\hline Parámetros & Media & 6,84 & 5,56 & 8,28 & 10,04 & 5,92 & 4,60 \\
\hline \multirow[t]{2}{*}{ Normales } & Desv. típica & 2,461 & 5,980 & 4,532 & 3,297 & 1,631 & 3,096 \\
\hline & Absoluta & 0,293 & 0,244 & 0,114 & 0,135 & 0,314 & 0,209 \\
\hline \multirow[t]{2}{*}{ Difer. Extremas } & S Positiva & 0,293 & 0,244 & 0,114 & 0,105 & 0,314 & 0,209 \\
\hline & Negativa & $-0,227$ & $-0,176$ & $-0,075$ & $-0,135$ & $-0,206$ & $-0,151$ \\
\hline \multicolumn{2}{|c|}{$\begin{array}{l}\text { Z de Kolmogorov- } \\
\text { Smirnov }\end{array}$} & 1,463 & 1,221 & 0,571 & 0,677 & 1,568 & 1,043 \\
\hline \multicolumn{2}{|c|}{$\begin{array}{l}\text { Sig. Asintótica: } \mathrm{p} \\
\text { (bilateral) }\end{array}$} & 0,028 & 0,101 & 0,900 & 0,750 & 0,015 & 0,227 \\
\hline
\end{tabular}

\section{G.C. Grupo control \\ G.E. grupo experimental EX: Examen}


Del cuadro se observa que la distribución de contraste es normal, excepto el valor del examen parcial y examen de entrada del grupo control, que sin embargo no afecta sobre la decisión de considerar los datos normalizados.

Tabla 2. Prueba de medias para comparar datos relacionados

\begin{tabular}{clcccc}
\hline & Media & N & $\begin{array}{c}\text { Desviación } \\
\text { típica. }\end{array}$ & $\begin{array}{c}\text { Error típico. de } \\
\text { la media }\end{array}$ \\
\hline \multirow{2}{*}{ Par 1 } & GC. Examen parcial & 6,84 & 25 & 2,461 & 0,492 \\
& GC. Examen final & 5,56 & 25 & 5,980 & 1,196 \\
\multirow{2}{*}{ Par 2} & GE. Examen parcial & 8,28 & 25 & 4,532 & 0,906 \\
& GE. Examen final & 10,04 & 25 & 3,297 & 0,659 \\
\multirow{2}{*}{ Par 3} & GC. Examen parcial & 6,84 & 25 & 2,461 & 0,492 \\
& GC. Examen entrada & 5,92 & 25 & 1,631 & 0,326 \\
Par 4 & GC. Examen final & 5,56 & 25 & 5,980 & 1,196 \\
& GC. Examen entrada & 5,92 & 25 & 1,631 & 0,326 \\
Par 5 & GE. Examen parcial & 8,28 & 25 & 4,532 & 0,906 \\
& GE. Examen entrada & 4,60 & 25 & 3,096 & 0,619 \\
Par 6 6 & GE. Examen final & 10,04 & 25 & 3,297 & 0,659 \\
& GE. Examen entrada & 4,60 & 25 & 3,096 & 0,619 \\
\hline
\end{tabular}

En este cuadro se presenta la comparación de medias de los exámenes parcial y final del grupo control y experimental, también se compara la media de los exámenes de ambos grupos con la prueba de entrada tomada a los alumnos al inicio de semestre 2015 - I.

Tabla 3. Prueba de Diferencia de Medias para comparar Datos Relacionados

\begin{tabular}{|c|c|c|c|c|c|c|c|c|}
\hline & & Difer & encias $r$ & lacionad & & & & \\
\hline Examen & & $\begin{array}{l}\text { Desv } \\
\text { iació }\end{array}$ & $\begin{array}{l}\text { Error } \\
\text { típico. } \\
\text { de la }\end{array}$ & $\begin{array}{r}95 \% \text { In } \\
\text { confian } \\
\text { dife }\end{array}$ & $\begin{array}{l}\text { rvalo de } \\
\text { a para la } \\
\text { encia }\end{array}$ & & & $\begin{array}{c}\text { Signific } \\
\text { ancia. }\end{array}$ \\
\hline & $\begin{array}{l}\text { Medi } \\
\text { a }\end{array}$ & $\begin{array}{c}\text { n } \\
\text { típica }\end{array}$ & media & Infer. & Super. & $\mathrm{t}$ & gl & bilateral \\
\hline $\begin{array}{l}\text { Par } 1 \text { GC. Examen parcial } \\
\text { GC. Examen final }\end{array}$ & 1,28 & 4,596 & 0,919 & $-0,617$ & 3,177 & 1,39 & 24 & 0,177 \\
\hline $\begin{array}{c}\text { Par } 2 \text { GE. Examen parcial } \\
\text { GE. Examen final }\end{array}$ & $-1,76$ & 4,893 & 0,979 & $-3,780$ & 0,260 & $-1,79$ & 24 & 0,085 \\
\hline $\begin{array}{l}\text { Par } 3 \text { GC. Examen parcial } \\
\text { GC. Examen entrada }\end{array}$ & 0,92 & 1,077 & 0,215 & 0,475 & 1,365 & 4,27 & 24 & 0,000 \\
\hline $\begin{array}{l}\text { Par } 4 \text { GC. Examen final } \\
\text { GC. Examen entrada }\end{array}$ & $-0,36$ & 5,041 & 1,008 & $-2,441$ & 1,721 & $-0,35$ & 24 & 0,724 \\
\hline $\begin{array}{l}\text { Par } 5 \text { GE. Examen parcial } \\
\text { GE. Examen entrada }\end{array}$ & 3,68 & 2,445 & 0,489 & 2,671 & 4,689 & 7,52 & 24 & 0,000 \\
\hline $\begin{array}{l}\text { Par } 6 \text { GE. Examen final } \\
\text { GE. Examen entrada }\end{array}$ & 5,44 & 3,959 & 0,792 & 3,806 & 7,074 & 6,87 & 24 & 0,000 \\
\hline
\end{tabular}


En este cuadro se presenta la diferencia de medias entre los exámenes parcial y final de cada grupo y de cada grupo con la prueba de entrada tomada a inicios del semestre 2015-I.

Tabla 4. Prueba de diferencias de medias de estadísticos de grupos independientes

\begin{tabular}{llcccc}
\hline & GRUPO & N & Media & $\begin{array}{c}\text { Desviación } \\
\text { típica. }\end{array}$ & $\begin{array}{c}\text { Error típico. de } \\
\text { la media }\end{array}$ \\
\hline \multirow{2}{*}{ Examen parcial } & Control & 25 & 6,8400 & 2,46103 & 0,49221 \\
& Experimental & 25 & 8,2800 & 4,53248 & 0,90650 \\
Examen final & Control & 25 & 5,5600 & 5,97969 & 1,19594 \\
& Experimental & 25 & 10,040 & 3,29747 & 0,65949 \\
Examen entrada & Control & 25 & 5,9200 & 1,63095 & 0,32619 \\
& Experimental & 25 & 4,6000 & 3,09570 & 0,61914 \\
\hline
\end{tabular}

En este cuadro se compara la diferencia de medias entre el grupo control y el grupo experimental, también se puede observar la media de ambos grupos en la prueba de entrada tomado en el semestre 2015 -I.

Tabla 5. Estadísticos descriptivos

\begin{tabular}{|c|c|c|c|c|c|}
\hline & $\mathrm{N}$ & Media & $\begin{array}{l}\text { Desviación } \\
\text { típica }\end{array}$ & Mínimo & Máximo \\
\hline Examen parcial GC & 25 & 6,84 & 2,461 & 5 & 13 \\
\hline Examen final GC & 25 & 5,56 & 5,980 & 0 & 18 \\
\hline Examen parcial GE & 25 & 8,28 & 4,532 & 0 & 18 \\
\hline Examen final $\mathrm{GE}$ & 25 & 10,04 & 3,297 & 4 & 15 \\
\hline Examen entrada GC & 25 & 5,92 & 1,631 & 4 & 10 \\
\hline Examen entrada GE & 25 & 4,60 & 3,096 & 0 & 11 \\
\hline
\end{tabular}

En este cuadro se observa las notas mínimas y máximas obtenidas en los exámenes parcial y final del grupo control y experimental. También de la prueba de entrada tomada a inicios del semestre 2015 - I. además se puede observar las medias del examen parcial y final de cada grupo. 


\section{TABLA 6. Prueba de Muestras Independientes}

Prueba de Levene

Para la igualdad

de Varianzas
Prueba t para la igualdad de medias

F $\quad$ Sig

gl sign. Difer, Error

$\mathrm{t} \quad \mathrm{g}$

$95 \%$ intervalo

de

(bilateral) de medias tipo $\begin{gathered}\text { confianza } \\ \text { Infer. Super. }\end{gathered}$

\section{Examen Parcial}

Con varianzas

Iguales

$\begin{array}{llllllll}4,824 & 0,033 & -1,40 & 48 & 0,169 & -1,44 & 1,03 & -3,51\end{array}$

0,63

Sin varianzas

Iguales

0,65

$$
\begin{array}{llllll}
-1,40 & 37,02 & 0,171 & -1,44 & 1,03 & -3,53
\end{array}
$$

\section{Examen Final}

Con varianzas

$$
1,73
$$

$$
\text { Iguales }
$$

$16,31 \quad 0,000$

$-3,28 \quad 48$

0,002

$-4,48$

$1,37 \quad-7,26$

Sin varianzas

Iguales

1,75

$\begin{array}{llllll}-3,28 & 37,36 & 0,002 & -4,48 & 1,37 & -7,25\end{array}$

\section{Examen de Entrada}

Con varianzas

2,73

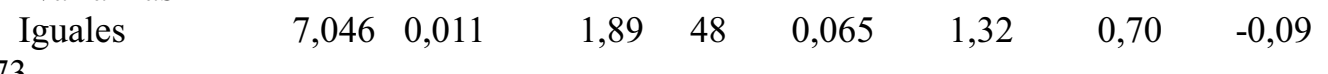

Sin varianzas

2,74

Iguales

$1,89 \quad 36,37 \quad 0,067$

1,32

$0,70 \quad-0,10$

En esta tabla se observa la comparación de varianzas entre el grupo control y grupo experimental para los exámenes parcial, final y la prueba de entrada tomados a inicios del semestre 2015 - I. además podemos observar el grado de significancia de cada elemento de prueba. 


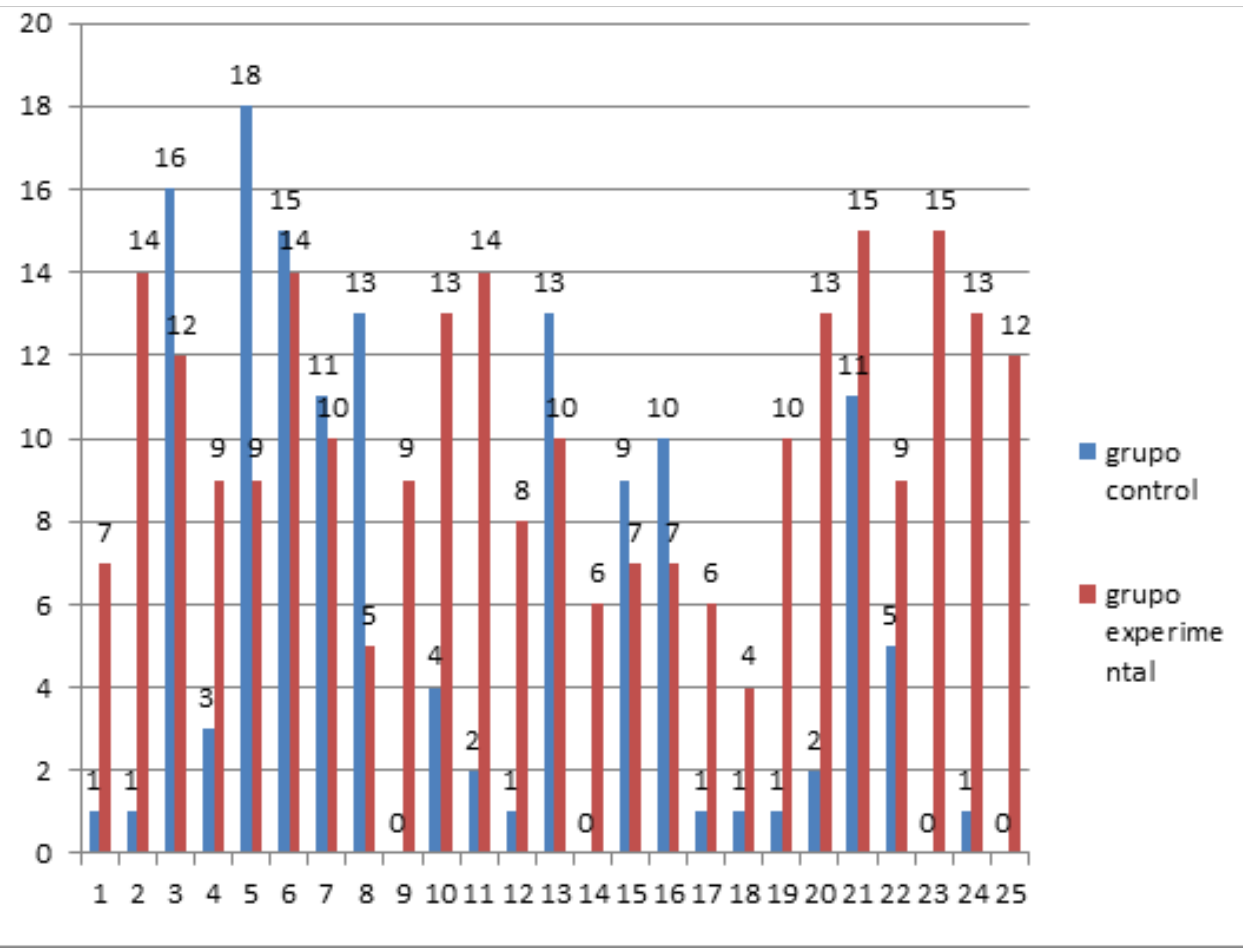

Figura 1. Resultado final del rendimiento académico del grupo Control y Experimental

\section{DISCUSION}

Mediante la prueba $\mathrm{t}$ de student comparamos las medias de datos relacionados (Tabla 2), ahí observamos que la media del examen parcial en el grupo experimental es de 8.28 es mayor que la media del grupo control que es 6.84, podría decirse que no existe homogeneidad de grupos, lo cual no es cierto como se observa en el anexo correspondiente a las actas parciales. (Primera practica calificada); esto sucede así debido a la naturaleza del experimento, que ha consistido en ir identificando mediante practicas calificadas las deficiencias de los alumnos y luego ir reforzando hasta llegar al examen parcial en donde ya se aprecia una diferencia en las medias de ambos grupos; que se acentúa mas en las medias del examen final en donde observamos una media de 10,04 para el grupo experimental contra 5.56 para el grupo control. También observamos en la prueba de entrada que se tomo a inicios del semestre 2015 - I a ambos grupos, que en ambos grupos ha existido aprendizaje pero con mayor notoriedad en el grupo experimental, existiendo mayor homogeneidad, en cuanto se observa menos diferencia entre el examen parcial, final y la prueba de entrada (8.28 y 10.04 contra 4.60), donde podemos observar que si hay una diferencia significativa en las notas de la prueba de entrada con el examen parcial y final lo que no sucede con el grupo control en donde la diferencia entre el examen parcial, final y la prueba de entrada es (6.84 y 5.56 contra 5.92) donde se observa que no existe mucha diferencia. 
En la Tabla 5 en el examen parcial el grupo control tubo una nota mínima de $5 \mathrm{y}$ una máxima de 13 y el grupo experimental una nota mínima de $0 \mathrm{y}$ máxima de 18 podemos observar que en los promedios el grupo control solo obtuvo 6.84 con una variación de 2.46, mientras que el grupo experimental tuvo una media de 8.28 con una variación de 4.53 de lo cual se puede observar que hay mejores notas en los alumnos del grupo experimental. En el examen final el grupo control tuvo una nota mínima de $0 \mathrm{y}$ máxima de 18 y el grupo experimental una nota mínima de 4 y máxima 15 . También observamos que la media del grupo control es de 5.56 con una variación de 5.98. Del grupo experimental la media fue de 10.04 con una variación de 3.297 de lo cual podemos observar que hay mas alumnos aprobados por lo tanto podemos decir que si existe una diferencia significativa en las notas de los alumnos del grupo experimental en relación al grupo control.

\section{CONCLUSIONES}

El rendimiento académico de los alumnos del grupo experimental conformado por los alumnos de la escuela de Ingeniería Agrícola matriculados en la asignatura de Matemática en el semestre 2015 - I , es significativamente mayor que la de los alumnos que conformaron el grupo control constituidos por los alumnos de la escuela profesional de Agronomía matriculados en el semestre 2015 - I.
Los calificativos obtenidos en el examen parcial y final presentan mejores notas en el grupo experimental en donde se sigue un método de evaluación sistematizado en comparación con el grupo control que se aplicó la forma tradicional.

La aplicación de un sistema de evaluación sistematizado mejoro significativamente el aprendizaje de los alumnos del grupo experimental comparado con los del grupo control en donde se aplico la forma tradicional de evaluación.

\section{REFERENCIAS BIBLIOGRAFICAS}

Antoni, Martin.2008. La evaluación de los aprendizajes. Construcción de instrumentos. Barcelona. Ediciones Octaedro S.L.

Bocanegra, Felix.1999. Bases Metodológicas de la Investigación Científica. Trujillo: Publi ciencias.

Brockbanck, Anne y Mcgill, Lan.2002. Aprendizaje Reflexivo en la Educación Superior. Madrid: Morata.

Arias, F. 2012. El proyecto de Investigación: Introducción a la metodología cientifica $6^{\text {ta }}$ Edición. Caracas. Editorial Epistome.

Hernández, Roberto. y Fernández, Carlos. 2000. Metodología de la Investigación. Distrito Federal: $2^{\text {da }}$ Ed. McGraw Hill. 
Moya, A.2013. Evaluación de los aprendizajes en matemáticas: una propuesta desde la educación matemática crítica. Caracas. Universidad Pedagógica Experimental Libertador.

Skovsmose, O.2012. Educação matemática crítica a questão da democracia ( $6 t^{a}$ edición). Campiñas. Papiras.

\section{CORRESPONDENCIA}

MSc. Cesar Augusto González

cgchiclayo15@hotmail.com 